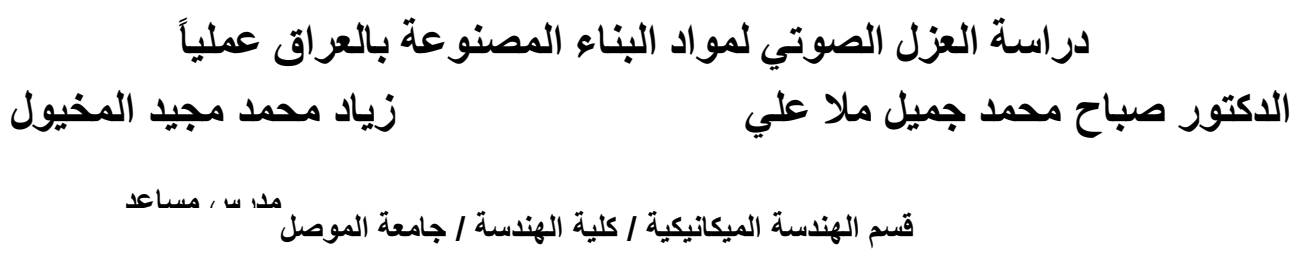

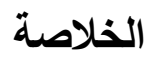

تم في هذا البحث در اسة العزل الصوتي لبعض المواد الغالب استخدامها في تثيبيد

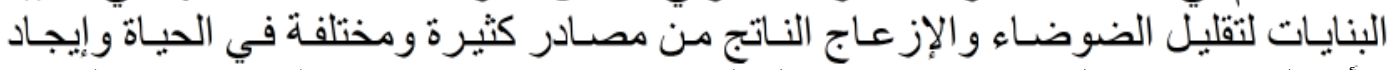

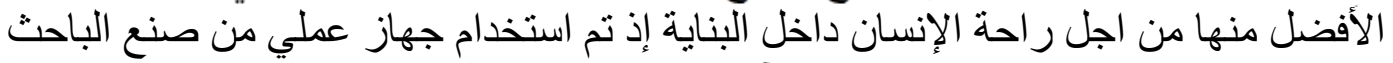

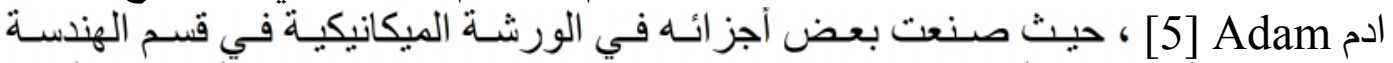

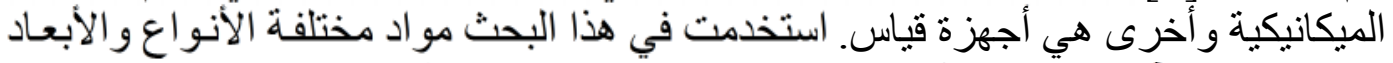

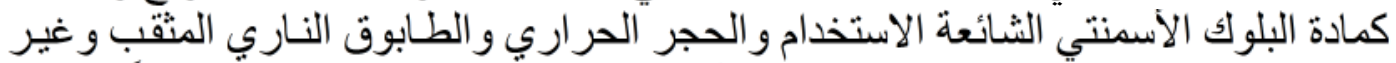

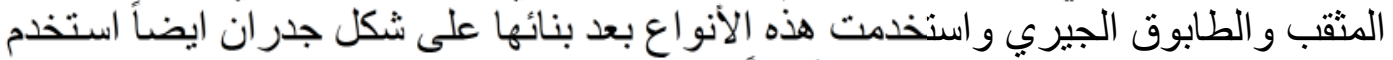

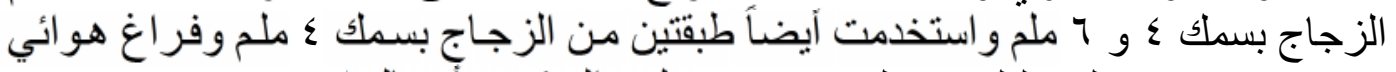

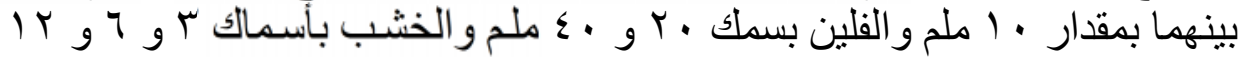

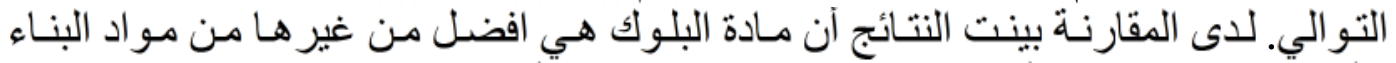

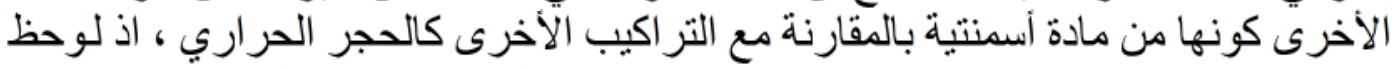

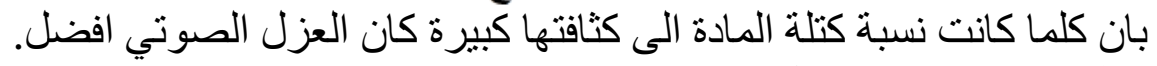

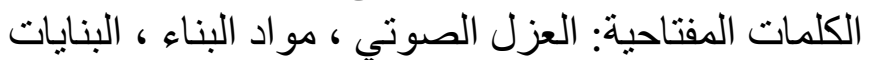

\title{
Experimental Study of the Sound Insulation for the Building Materials in Iraq
}

\author{
Dr. S.M.J. Ali \\ Professor \\ Ziad M. Al-Makhyoul \\ Department of Mechanical Engineering -University of Mosul - \\ Iraq
}

\begin{abstract}
The study of acoustic insulation for some materials which were used in most building is investigated in this paper to reduce the noise and distribance which generated from the different sources. The object is to the best comfort to the human. A practical device is made by author and is used in this work. Some of these parts are made in the
\end{abstract}


workshop of mechanical engineering and the other apparatus measurements. The concrete block, thermostone and fire block are used as walls for buildings. The glass was used with thick $(4,6) \mathrm{mm}$ and two layer of glasses with thick $4 \mathrm{~mm}$ with $10 \mathrm{~mm}$ gap, thirospore and wood are used with thick $(20,40) \mathrm{mm}$ and thickness of $(3,6,12) \mathrm{mm}$ respectively. The comparison between above material shows that the concrete block is the best material being made from the concrete, as a comparison with other constructions. It was notice when the ration of mass to density for any material was high, the sound insulation for this material becomes excellent.

Key words: Sound insulation , building materials , buildings

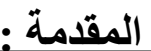

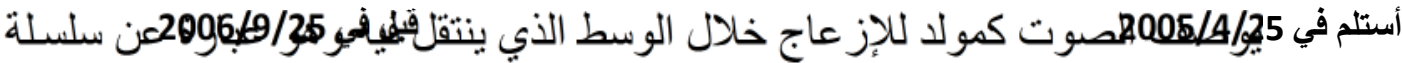

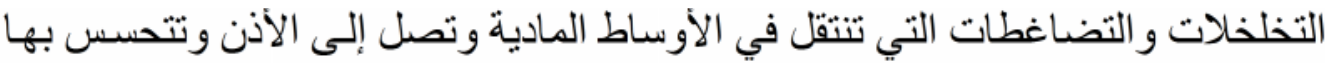

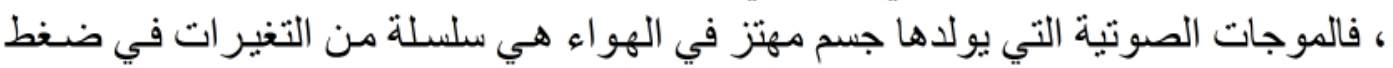

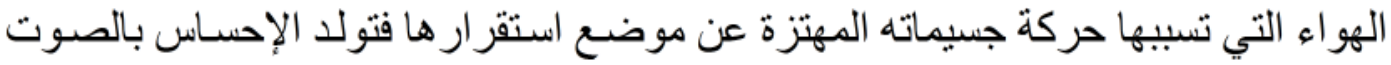

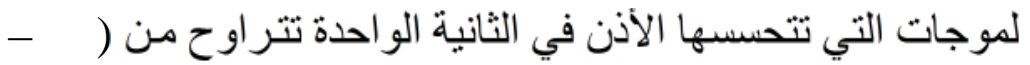

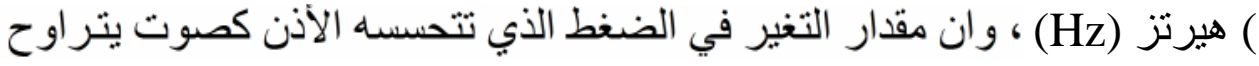

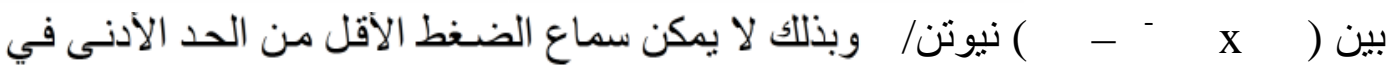

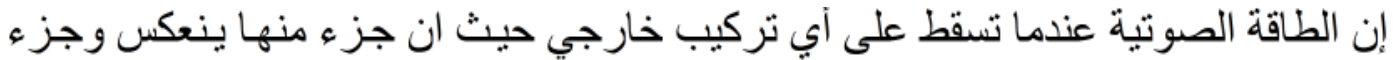

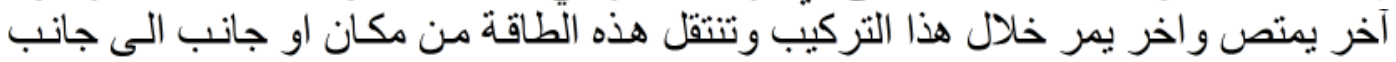

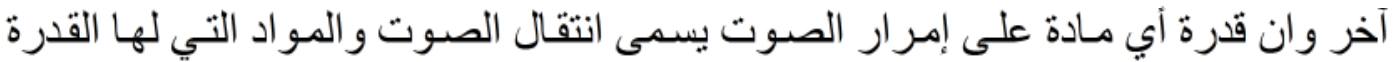

إن المعنى الفيزياوي لقيمة العزل الصوتي لحاجز ما هي النسبة المتوقفة على قدرة

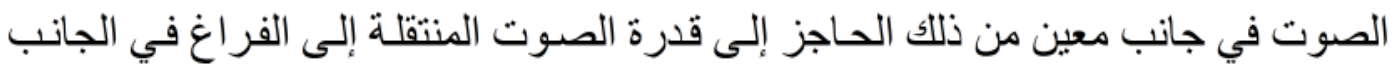

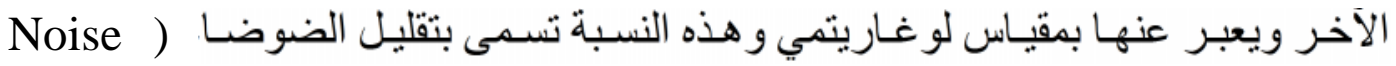

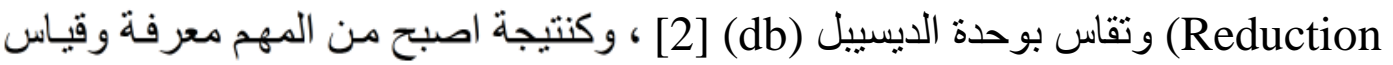

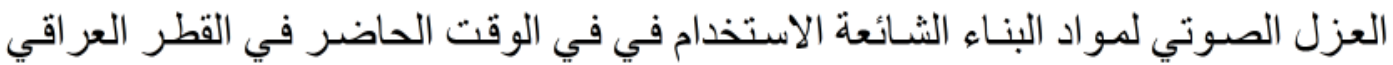

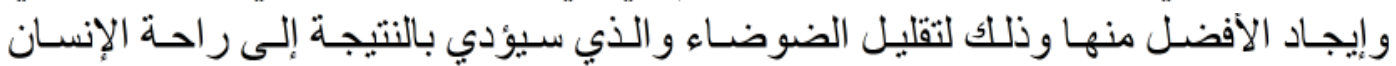

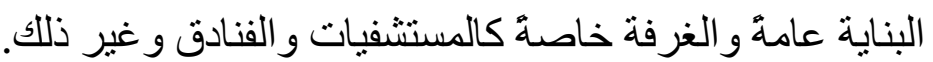


[3] Hiroo

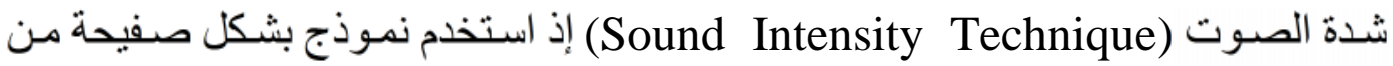

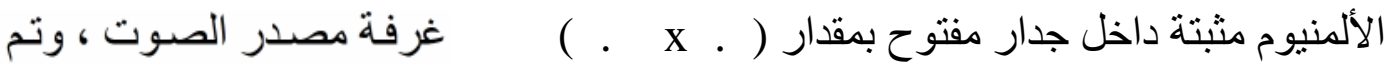

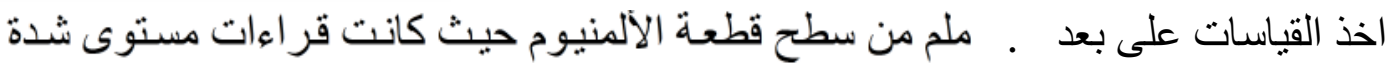

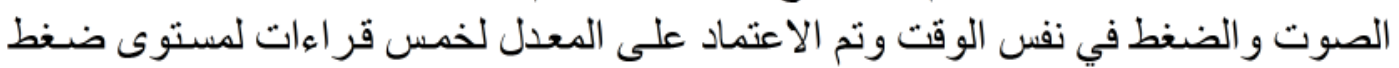

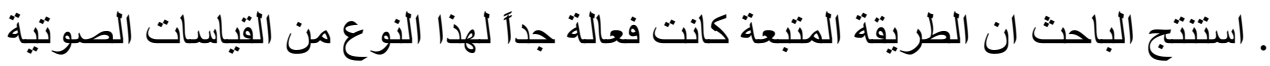

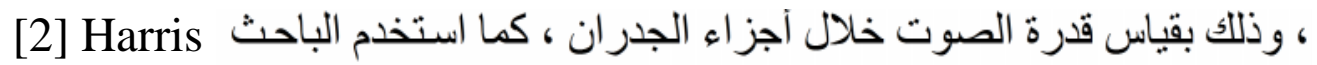

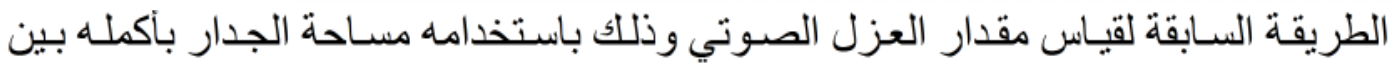

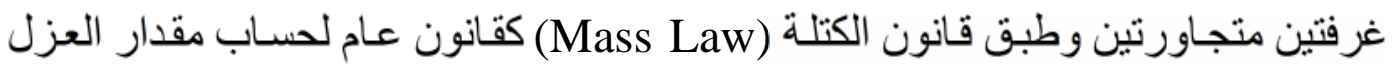

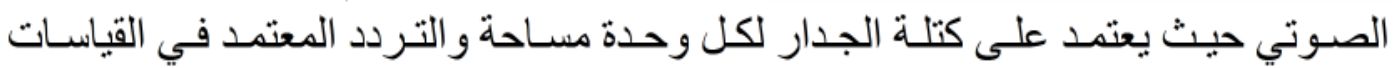

$\mathrm{TL}=20 \log (\mathrm{f} * \mathrm{~m})-48$

(..(1)

TL مقدار العزل الصوتي بوحدة الديسييل

$\mathrm{m}=1$

f =(Hz ) التردد بوحدة الهيرتز

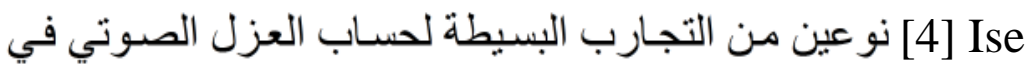

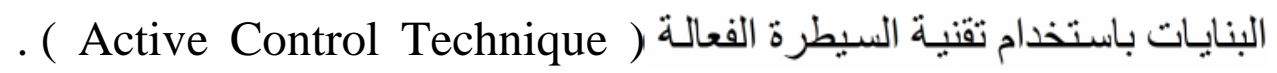

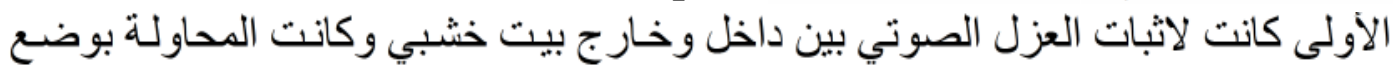

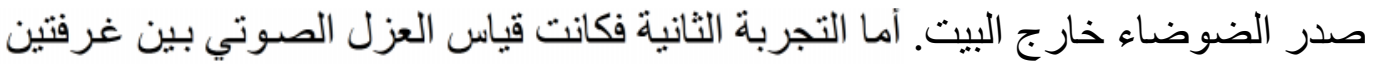

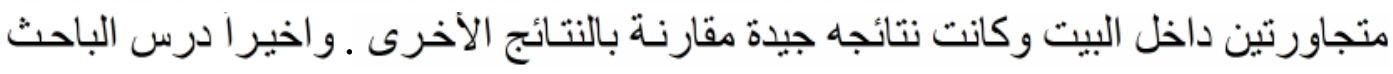

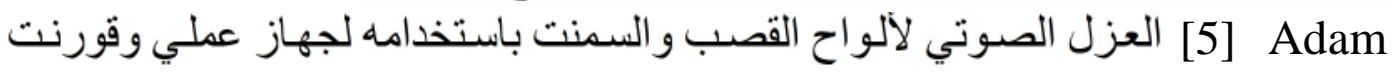

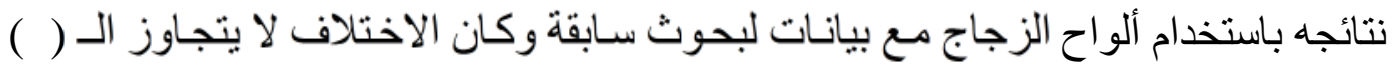
ديسييل لمدى كامل من الذبذبات. 


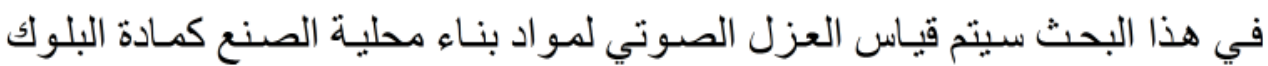

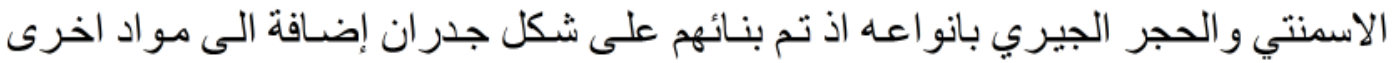
و الفلين اذ سيتم ايجاد الافضل منها من اجل راحة النا الانسان داخل البنايات.

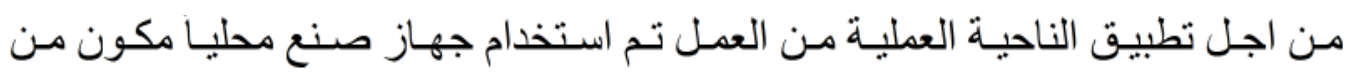

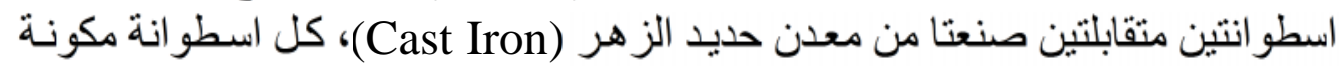

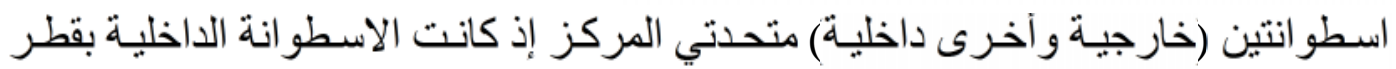

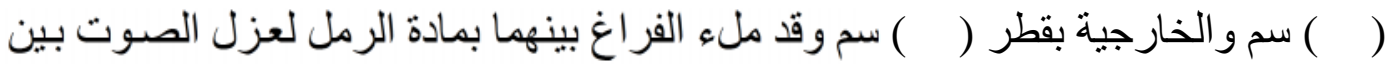

داخل الاسطو انة و المحيط الخارجي كما في الثكل ( ) ).

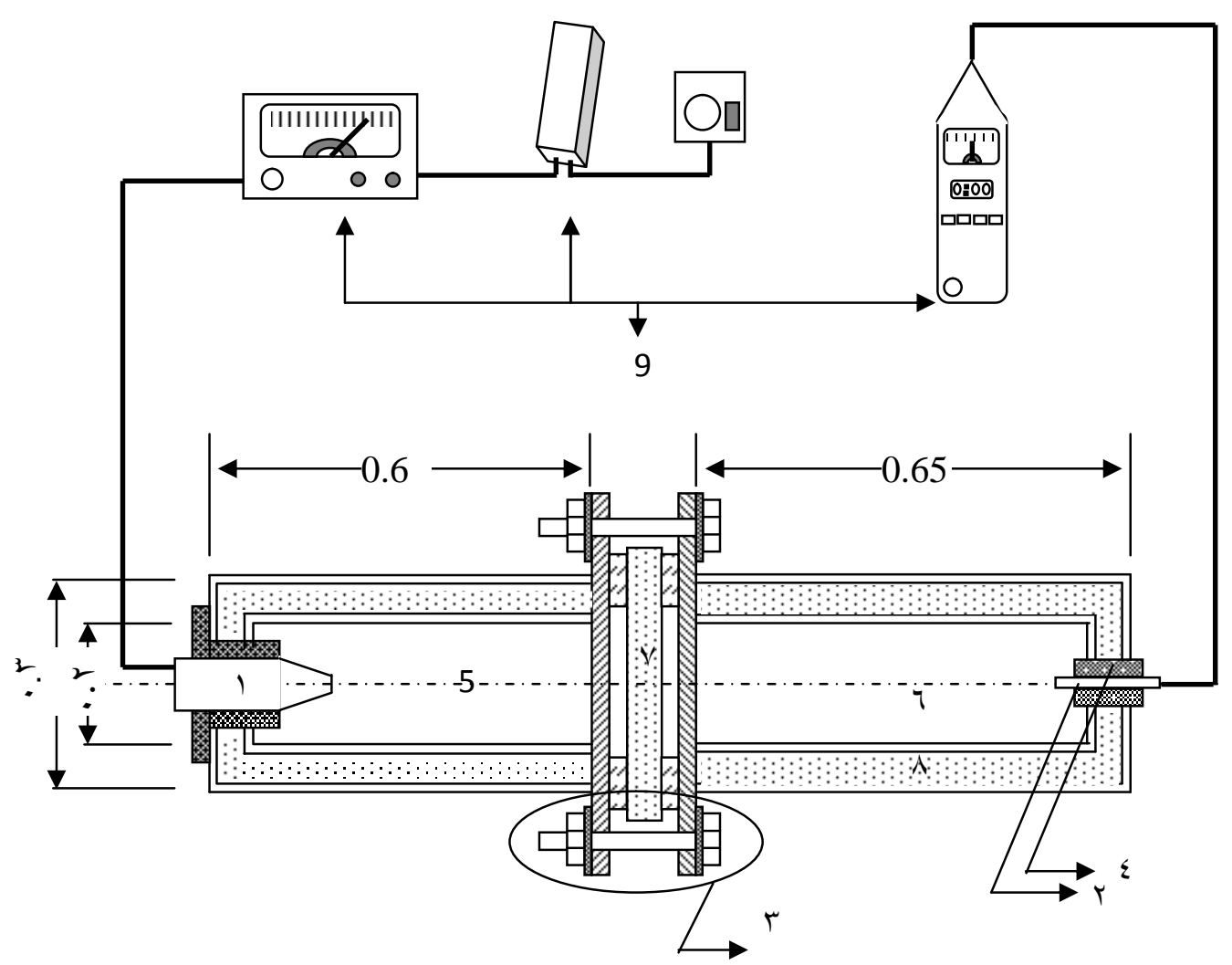

الثكل ( (1) مخططا للجهاز المستخدم وأجهزة القياس المستخمة 
(Loud speaker)

(Microphone)

ـ البر اغي و الو اشرات المطاطية (Rubber washer and nuts)

ـ ـ المادة الاسفنجية (Sponge material)

- حيز المصدر ( Source Zone).

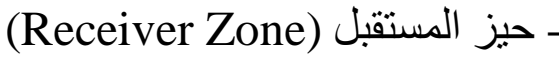

ـ المادة المر اد قياس عزلها الصوتي (Specimen)

(Sand)

ـ أجهزة القياس (Measurement Instruments) 
الاسطو انة الأولى طولها ( ) سم وتسمى حيز المصدر (Source Zone) و الثانية

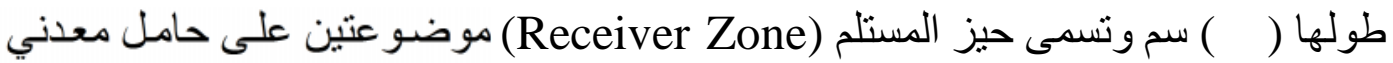

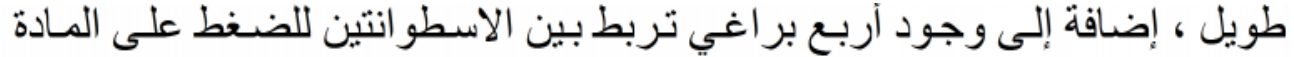

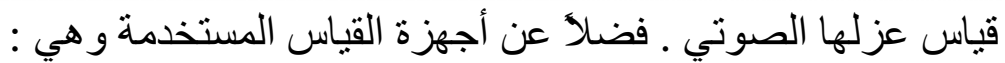

$$
\begin{aligned}
& \text {.(Amplifier) -1 } \\
& \text { (Microphone) - التحويل الموجات الصوتية إلى موجات كهربائية. } \\
& \text { r- ب- مجز القدرة (Power Supply). } \\
& \text { ـ - جهاز مسجل ضغط الصوت (Sound Level Meter). }
\end{aligned}
$$

اما طريقة اجر اء التجربة فستوضح باختصار ، اذ تربط في البداية الدائرة الكهربائية

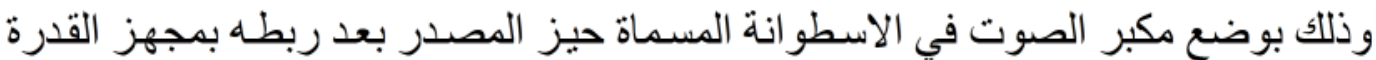

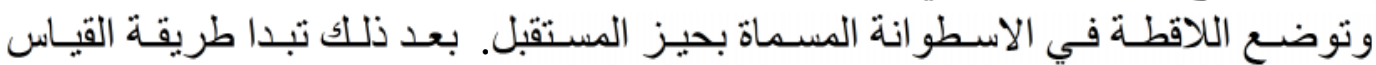
وكخطوة أولى ورئيسية تم اخذ القر اءات عند الات عدم وجود أي مـادة بين الاسطو انتين إذ تم

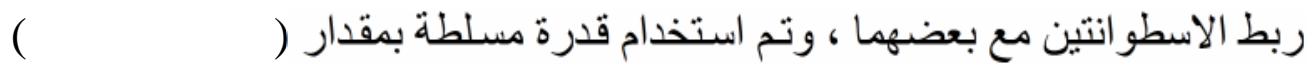

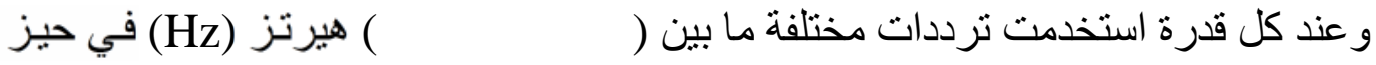
المصدر واستقبلت هذه الترددات في حيز المستقبل عن طريق التلاقطة التي من وضيفتها

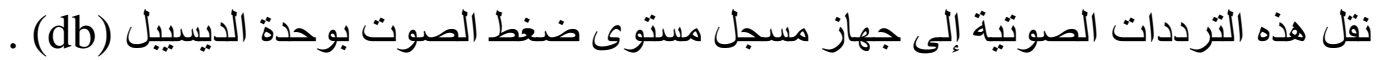

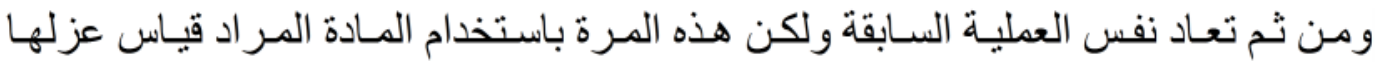
الصوتي و الفرق بين العمليتين هو مقدار العزل الصوني الصني للمادة .

استخدم في هذا البحث عدة مو اد كالبلوك الأسمنتي و الثرموستون و الطابوق بانو اعها

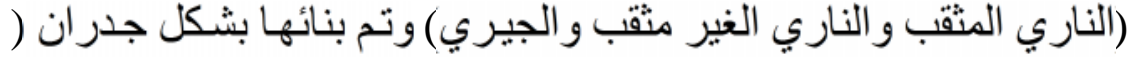

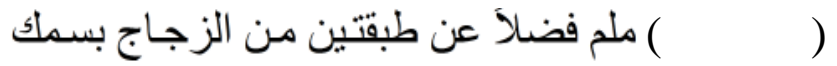

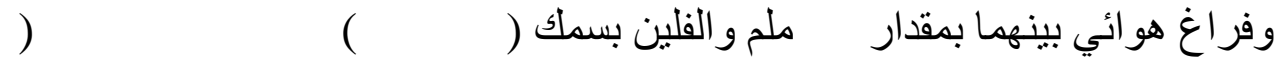

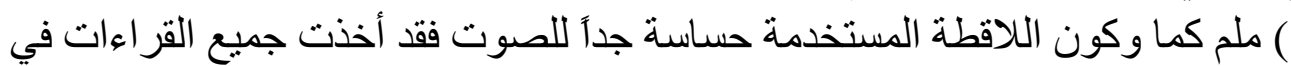

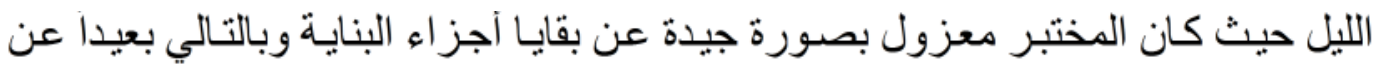




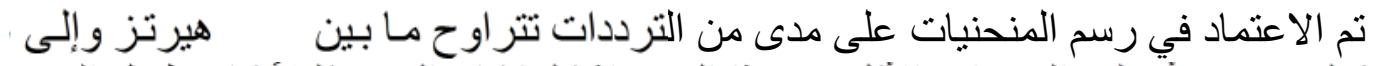

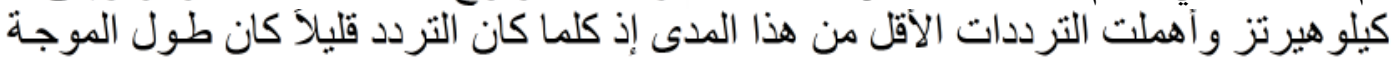

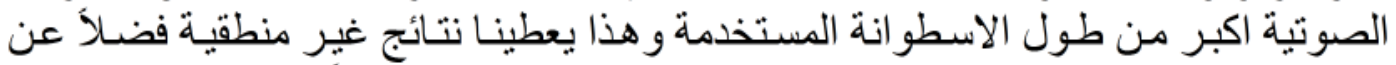
الحصول على توزيع متجانس للصوت في كل من الاسطو انتين فضـلاً عن كون المنحنيات

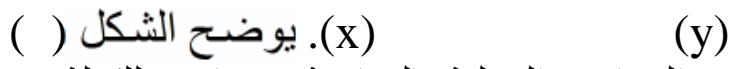

النتائج التي تم الحصول عليها لمادة البلوك من التجارب العملية بالمقارنة مع قانون للكتلة في

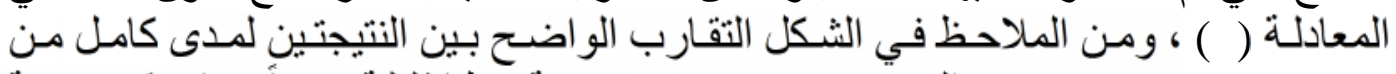

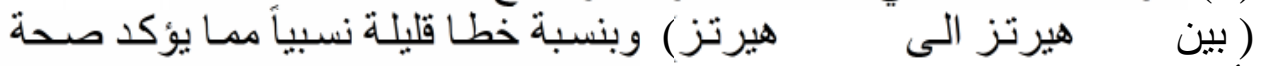

النتائج المأخوذة من الجهاز المستخدم ـ لهين

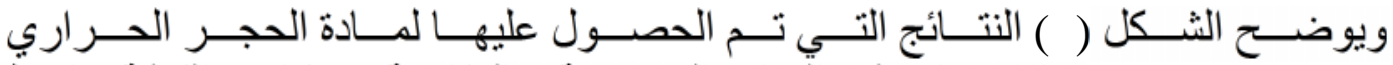

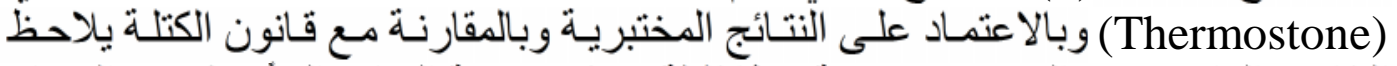

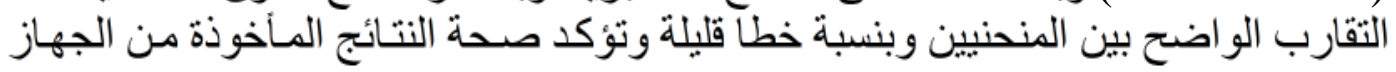

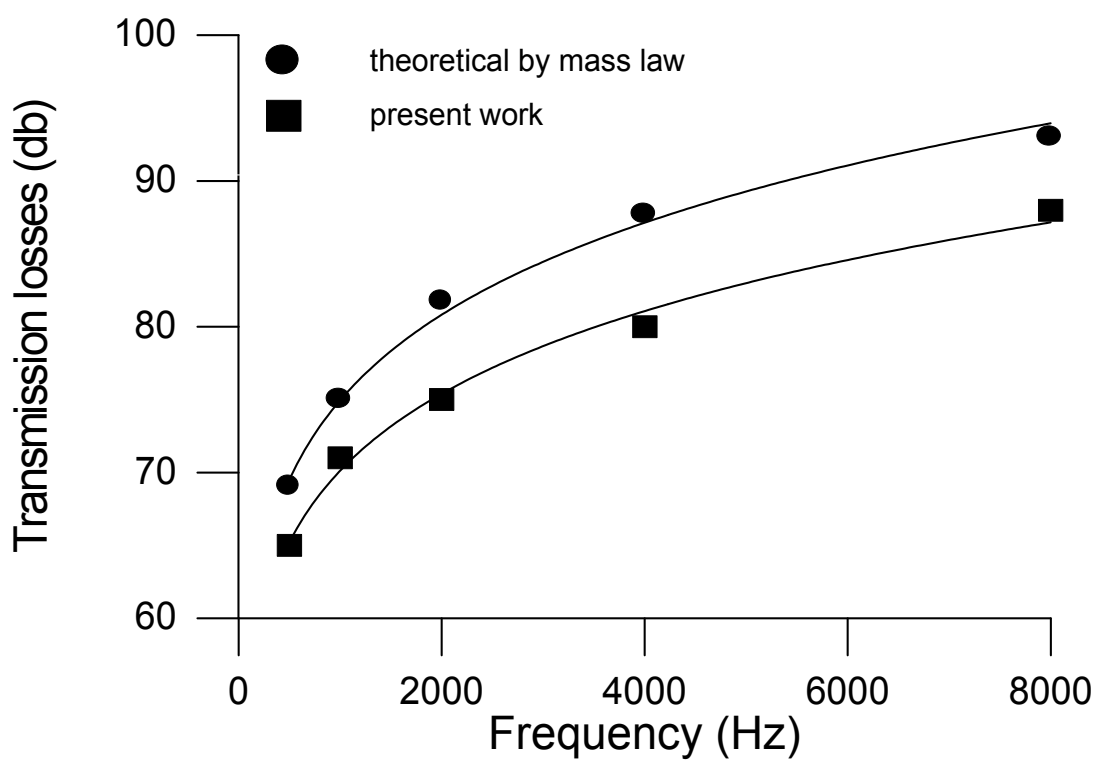

شكل (r) يوضح مقارنة العزل الصوتي بين النتائج العطلية الحالية مع النتائج النظرية باستخدام

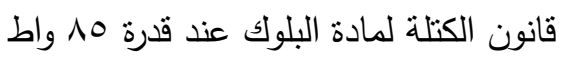




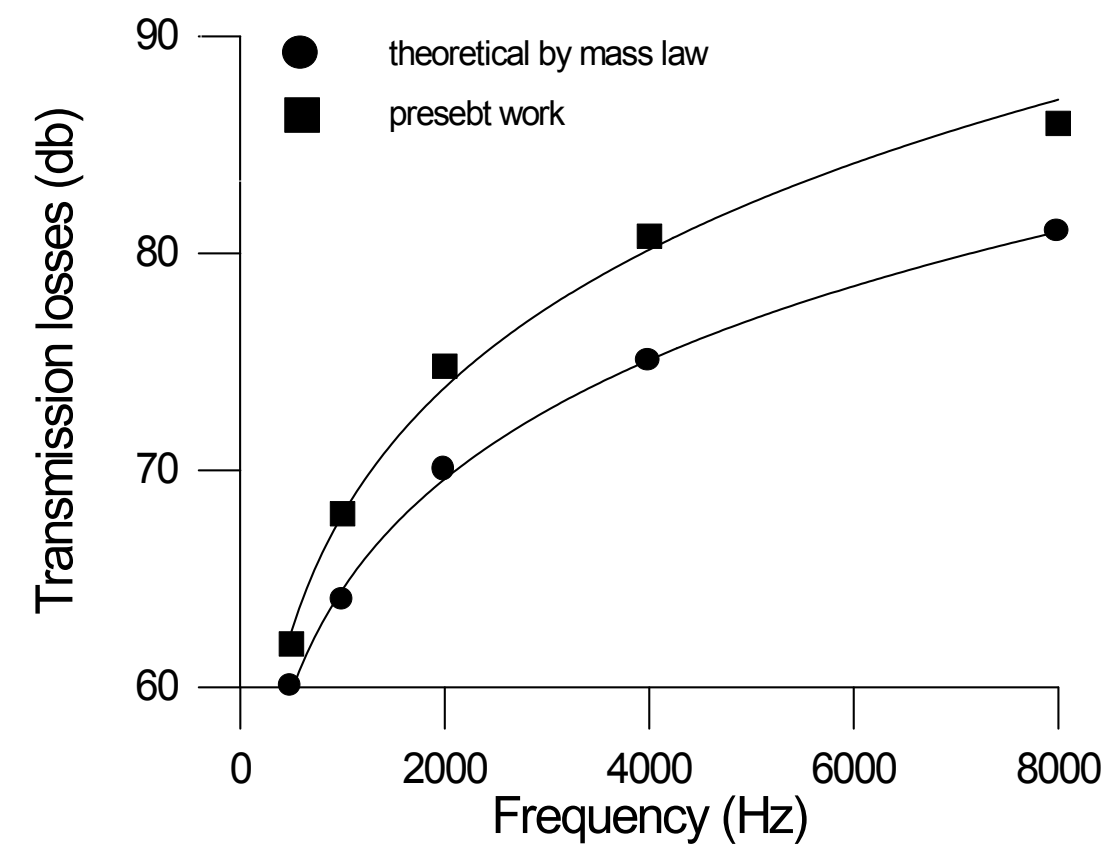

شكل (r) يوضح مقارنة العزل الصوتي بين النتائج العملية الحالية مع النتائج النظرية باستخدام

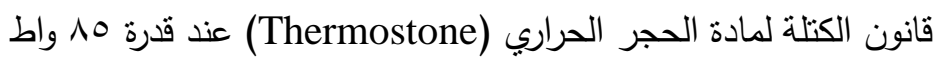

يوضح الثنكل ( ) مقارنة بين مادتي البلوك و الحجر الحر اري بعد ان تم التاكد من نتائجهما

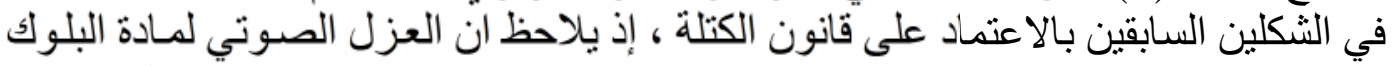

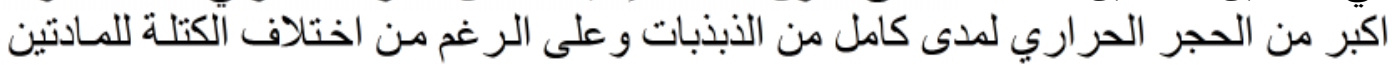

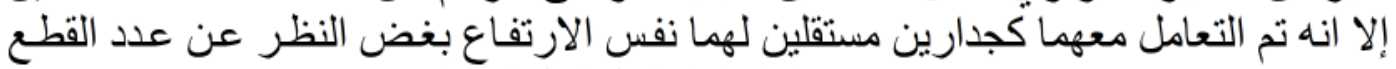

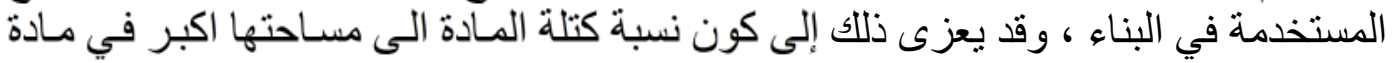

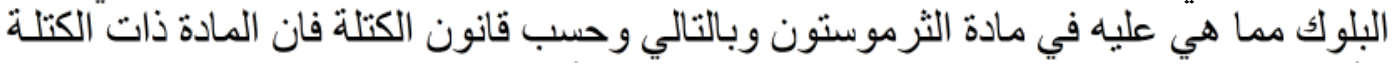
: [5]

\begin{tabular}{|l|l|}
\hline الكتلة (كفم/م (المادة & \\
\hline & \\
\hline & \\
\hline
\end{tabular}

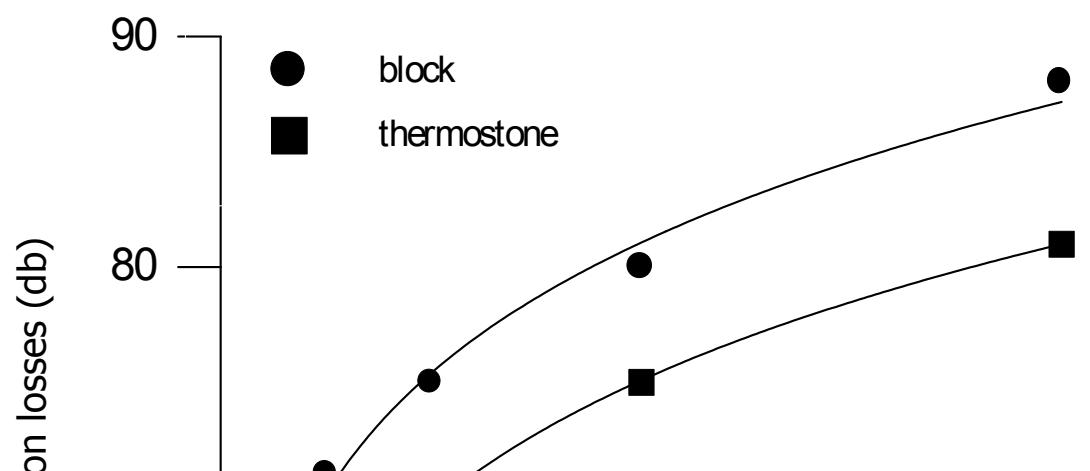




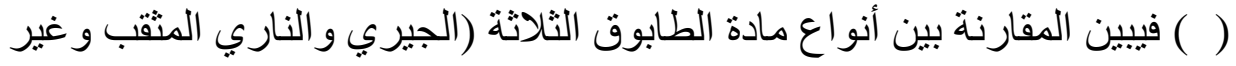

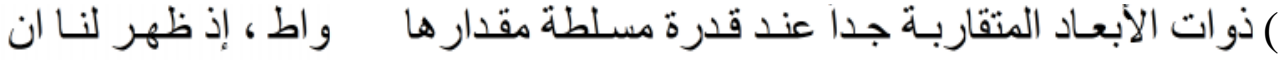

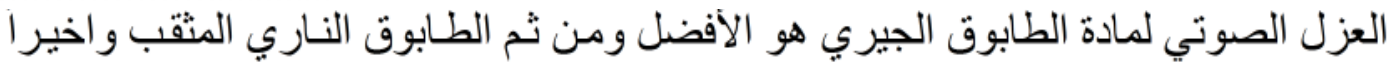

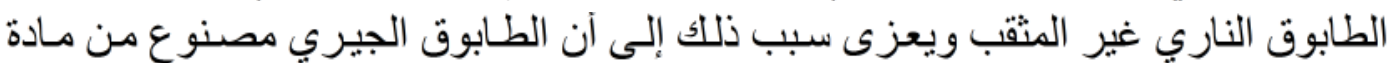

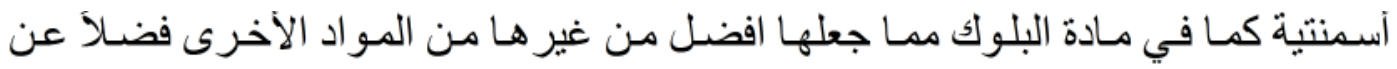

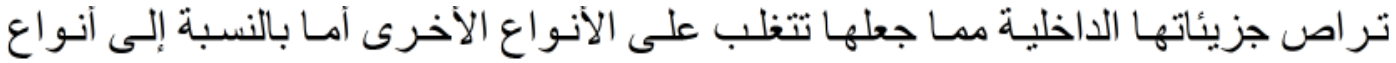

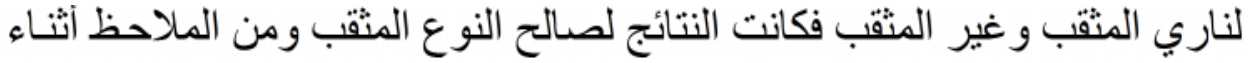

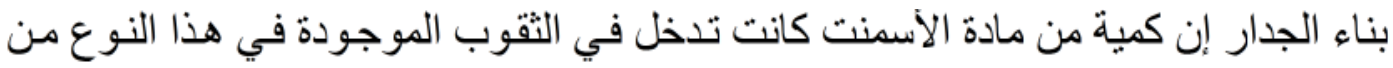
الطابوق مما جعل عزلها الصوتي افضل من النوع الثاني الغير مثنب.

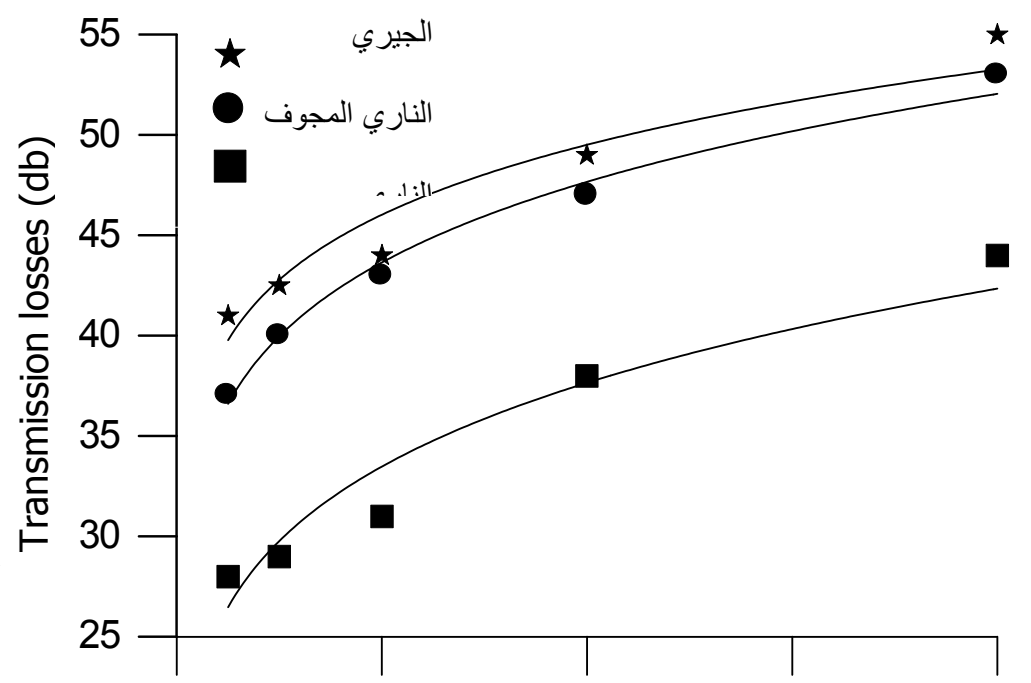




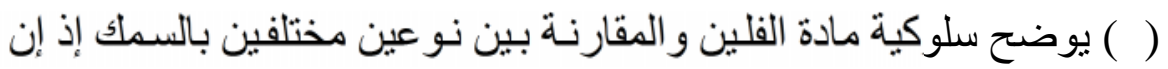

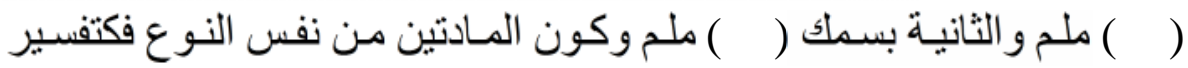
طبيعي ان المادة ذات السمك الأكبر تمتلك عزل صوتي اكبر من نظير تها ذات السمك الأقل

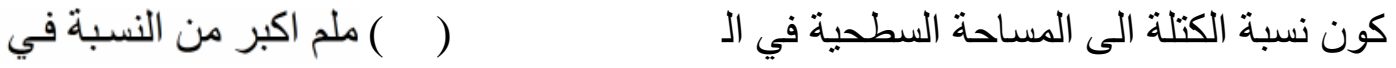

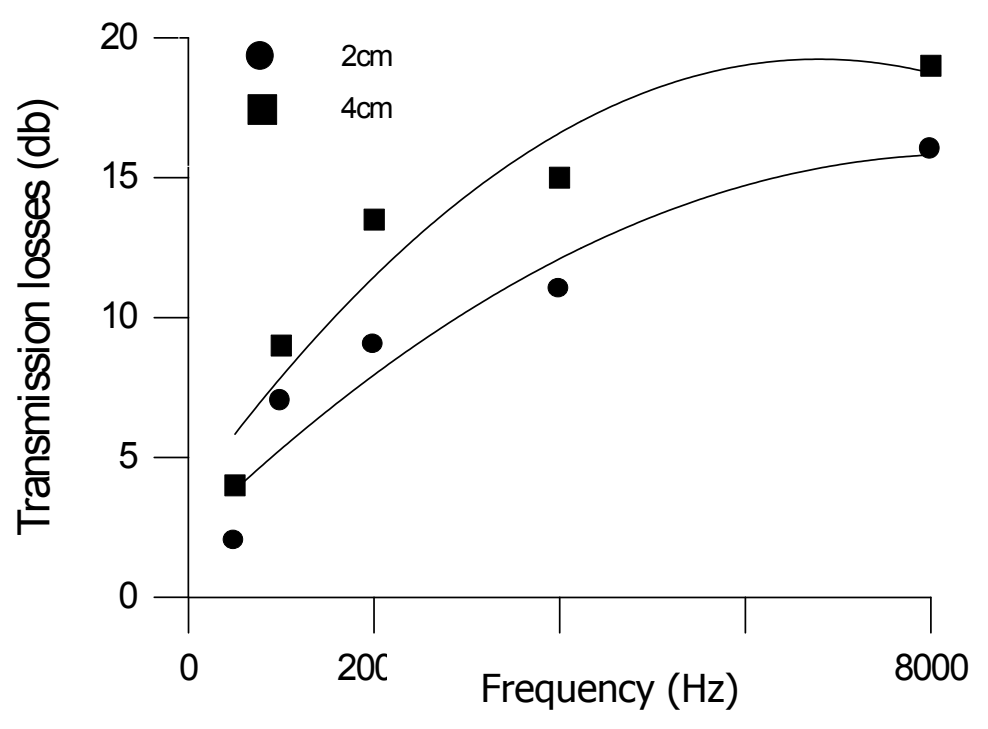


يبين الثكل ( ) مقارنة بين ثلاثة أنشال لمـادة الزجاج الاول بسمك

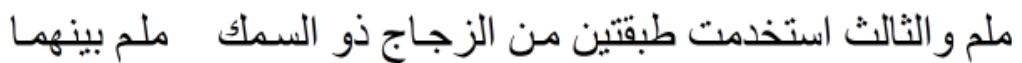

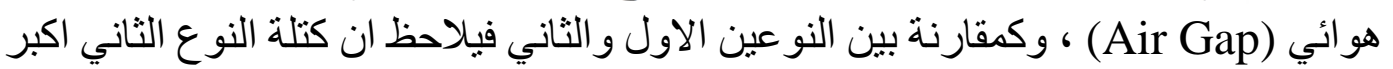

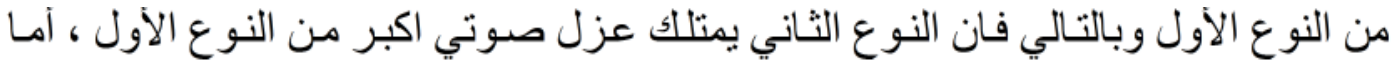

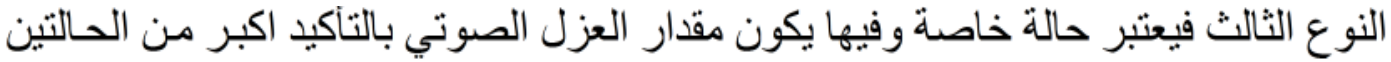

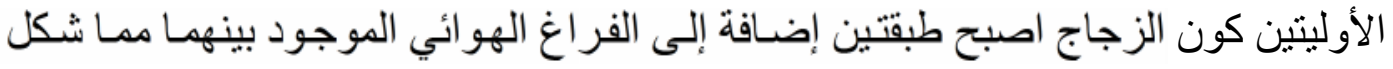
عاز لا كبير ا امام اختر اق الصوت اصني لهذا التركيب.

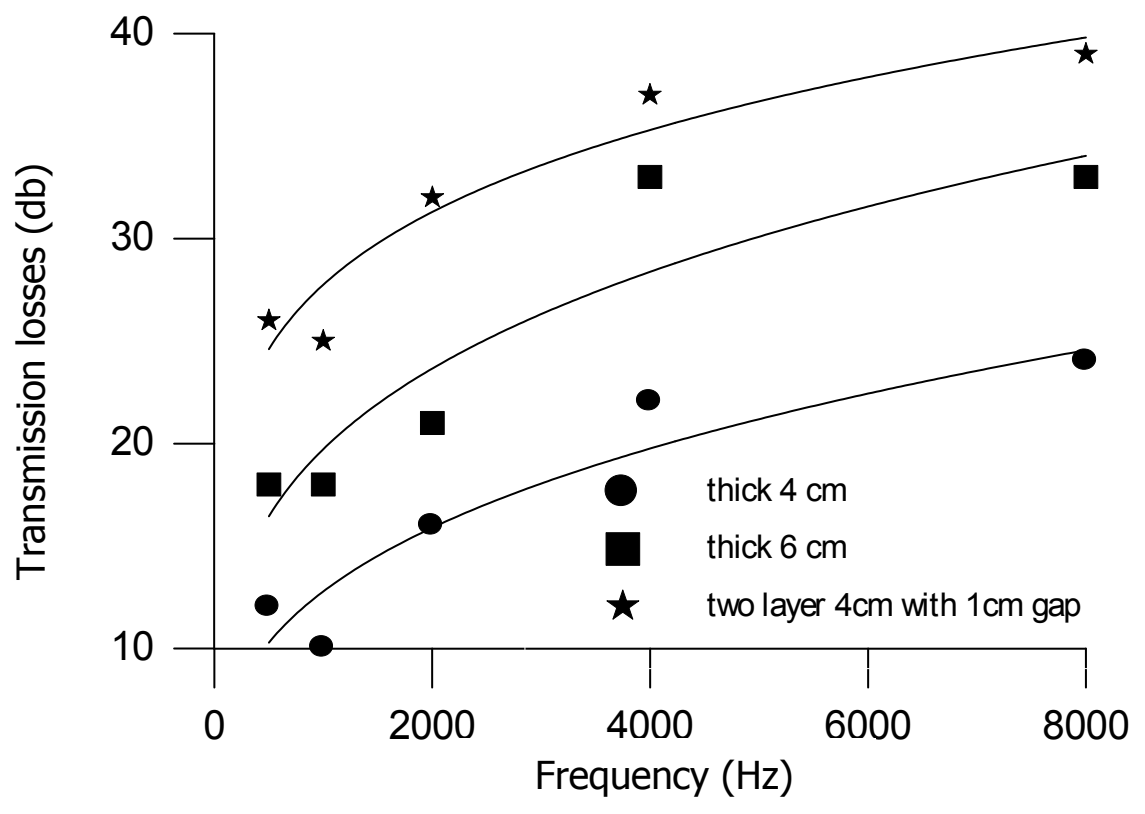

شكل (V) ييين قيم العزل الصوتي لمادة الزجاج عند ثلاثة حالات مختلفة وعند قدرة 10 واط 


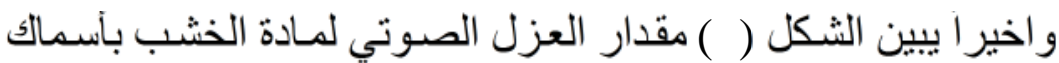

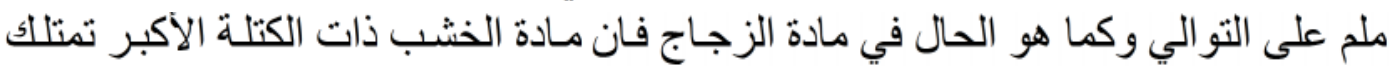

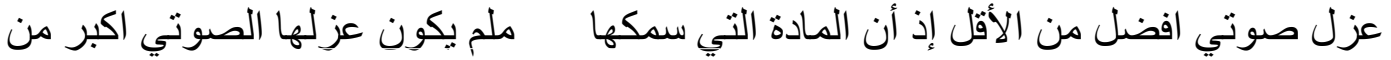
المادة التي هي بسمك

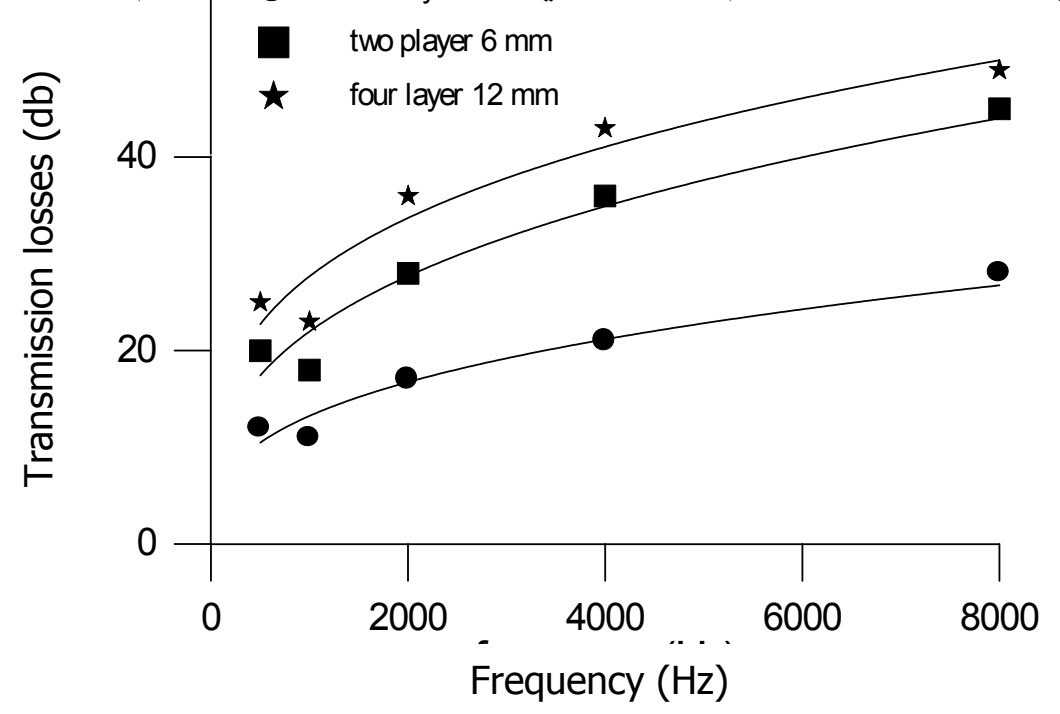

شكل (^) يبين قيم العزل الصوتي لمادة الخشب لثناثة اسماك مختلفة عند قدرة م^ و اط 


$$
\text { يلاحظ إن نسبة الخطأ لم تتجاوز }
$$

-بعد المقارنـات أعـلاه ، وجدنا آن المـادة التـي تكون نسبة كتلتها إلى مســاحتها

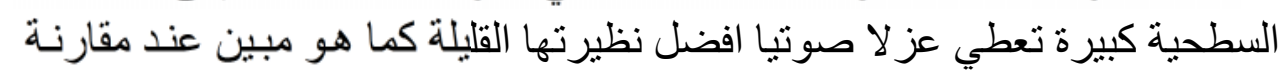

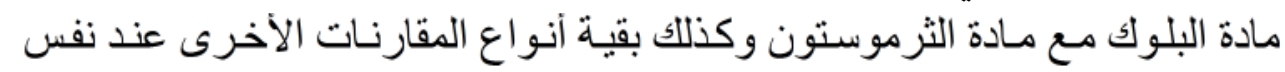

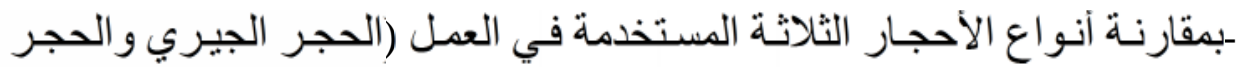

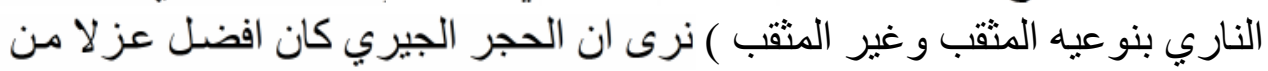

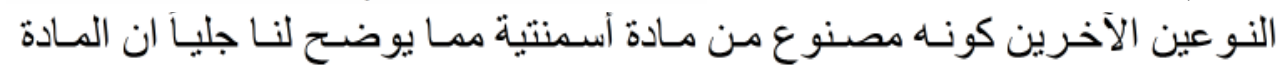
الأسمنتية تمنلك عزل صوتي الإنس كن من غير ها من المو اد.

\section{References}

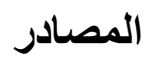

1- Kormer, A., Building Materials and Components, Mir Publisher, Moscow, 1974.

2- Harris, C.M. Handbook, of Noise Control, McGraw-Hill, U.S.A.,1979.

3- Hiroo, Y., Hideki, T. and Kiyotern, I., “ Application of Sound Intensity Measuring Technique to Sound Insulation “, Institute of Industrial Scince, University of Tokyo, roppongi 7-22-1, MinotaKu, Japan, 1983. 
4- Ise, S., Yano, H. and Tachibana, H., " Application of Active Control to Sound Insulation of Building walls "Institute of Industrial Scince, University of Tokyo, roppongi 7-22-1, MinotaKu, Japan, 1991.

5- Adam. S., K., "An Experimental and Theoretical Studies of Thermal and Acoustical Properties of Reed-Cement Boards ", Ph.D., Thesis, University of Mosul, 1997. 